\title{
Entangled Inputs Cannot Make Imperfect Quantum Channels Perfect
}

\author{
F. G. S. L. Brandão, ${ }^{1}$ J. Eisert, ${ }^{2,3}$ M. Horodecki, ${ }^{4}$ and D. Yang ${ }^{5}$ \\ ${ }^{1}$ Departamento de Física, Universidade Federal de Minas Gerais, Belo Horizonte 30123-970, Brazil \\ ${ }^{2}$ Institute of Physics and Astronomy, University of Potsdam, 14476 Potsdam, Germany \\ ${ }^{3}$ Dahlem Center for Complex Quantum Systems, Freie Universität Berlin, 14195 Berlin, Germany \\ ${ }^{4}$ Institute of Theoretical Physics and Astrophysics, University of Gdańsk, 80-952 Gdańsk, Poland \\ ${ }^{5}$ Laboratory for Quantum Information, China Jiliang University, Hangzhou, Zhejiang 310018, China
}

(Received 26 November 2010; published 10 June 2011)

\begin{abstract}
Entangled inputs can enhance the capacity of quantum channels, this being one of the consequences of the celebrated result showing the nonadditivity of several quantities relevant for quantum information science. In this work, we answer the converse question (whether entangled inputs can ever render noisy quantum channels to have maximum capacity) to the negative: No sophisticated entangled input of any quantum channel can ever enhance the capacity to the maximum possible value, a result that holds true for all channels both for the classical as well as the quantum capacity. This result can hence be seen as a bound as to how "nonadditive quantum information can be." As a main result, we find first practical and remarkably simple computable single-shot bounds to capacities, related to entanglement measures. As examples, we discuss the qubit amplitude damping and identify the first meaningful bound for its classical capacity.
\end{abstract}

DOI: 10.1103/PhysRevLett.106.230502

PACS numbers: 03.67.Hk, 03.67.Ac

How much information can one transmit reliably through a quantum channel such as a telecommunication fiber? This basic question is, despite much progress in recent years [1-6], still surprisingly wide open. Some suitable encoding and decoding is necessary, needless to say, but the optimal achievable rates can still not be expressed in a computable closed form. For classical information, the hope that the single-shot capacity would be sufficient to arrive at that goal was corroborated by many examples of channels for which this is in fact true [2]. Alas, it was finally found to be unjustified with the celebrated result [1] on the nonadditivity of several quantities that are in the center of interest in quantum information science [3-5]. In particular, entangled inputs help and do increase the classical information capacity. Thus the question of finding capacities of quantum channels is more complicated than what one might have anticipated. In the case of quantum information transmission, a similar situation has been known to be true already for a long time: In general, to attain quantum capacity, one must regularize the single-shot expression, given by the coherent information [7].

To contribute to fixing the coordinate system of channel capacities, this insight begs for a resolution of the following question: To what extent can entanglement help then? Is the mentioned result rather an academic observation, manifesting itself in small violations of additivity in high physical dimensions? An interesting question in this context is the following: Can suitably entangled inputs render noisy quantum channels to take their maximum possible capacity or make them even perfect? This would be the other extreme, where the nonadditivity serves as a resource to overcome the noisiness of channels.
In this work, we answer this question to the negative: For all quantum channels, no matter how elaborate the entangled coding over many uses of the channel might be, one can never achieve the maximum possible capacity if this is not already true on the single-shot level. This observation holds true both for the classical as well as the quantum capacity.

We show this by introducing new upper bounds to these capacities which can be evaluated on the single-shot level and are computable, which constitute a main result of this work. We connect questions of capacities to those of entanglement measures of systems and their environments. These bounds are useful in their own right, which will be shown by means of an example of an amplitude-damping channel.

Notation and setting.-We start our discussion by fixing the notation and clarifying some basic concepts. We consider general quantum channels of arbitrary finite dimension $T: \mathcal{S}\left(\mathbb{C}^{d}\right) \rightarrow \mathcal{S}\left(\mathbb{C}^{d}\right)$, modeling any general noisy quantum evolution. $T$ is hence an arbitrary tracepreserving completely positive map. Such a channel can always be written in terms of a Stinespring dilation as

$$
T(\rho)=\operatorname{tr}_{E}\left(U \rho U^{\dagger}\right),
$$

labeling the input by $A$, associated with the Hilbert space $\mathbb{C}^{d}$, the output by $B$, and the environment by $E$, equipped with Hilbert spaces $\mathbb{C}^{d}$ and $\mathbb{C}^{d_{\text {env }}}$, respectively. $U$ is an isometry mapping the input on $A$ onto a quantum state on $B$ and $E$; see Fig. 1 .

The classical information capacity, or short classical capacity, of a quantum channel is the rate at which one can reliably send classical information. It is related to the Holevo- $\chi[8]$ or the single-shot classical capacity: 


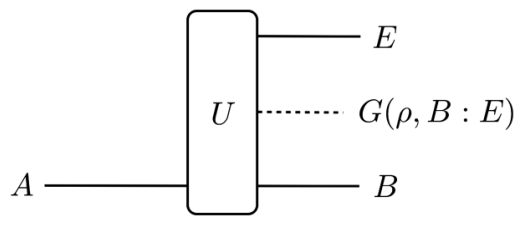

FIG. 1. Upper bound to the classical information capacity in terms of entanglement measures between the output and its environment in a dilation of the quantum channel.

$$
\chi(T)=\max \left\{S\left[\sum_{j} p_{j} T\left(\rho_{j}\right)\right]-\sum_{j} p_{j} S\left[T\left(\rho_{j}\right)\right]\right\},
$$

where the maximum is taken over probability distributions and states, as the asymptotic regularization

$$
C(T)=\limsup _{n \rightarrow \infty} \frac{\chi\left(T^{\otimes n}\right)}{n} .
$$

The trivial maximum value that the capacity can possibly take is the maximum output entropy of the channel:

$$
C(T) \leq \max _{\rho} S[T(\rho)]=S_{\max }(T) .
$$

We will say that whenever this bound is saturated, so when $C(T)=S_{\max }(T)$, the channel has maximum capacity, giving rise to the maximum that is trivially possible. Of course, this notion includes the situation of a perfect quantum channel that has a maximum output entropy of $C(T)=S_{\max }(T)=\log _{2}(d)$.

The quantum capacity of a quantum channel, in turn, is related to the rate at which one can reliably send quantum information through a quantum channel. Writing

$$
Q_{1}(T)=\max _{\Psi}\left[S\left(\omega_{B}\right)-S\left(\omega_{E}\right)\right],
$$

calculated in the state $\omega=|\phi\rangle\langle\phi|, \Psi=| \psi\rangle\langle\psi|$, as a state on $R, B$, and $E$, and where $|\phi\rangle:=U|\psi\rangle$, with $U$ being again the isometry of $T$, mapping $A$ to $B$ and $E$, and $|\psi\rangle$ being a state on $R$ and $A$. The quantum capacity is then

$$
Q(T)=\limsup _{n \rightarrow \infty} \frac{Q_{1}\left(T^{\otimes n}\right)}{n},
$$

again, referred to as maximum if $Q=S_{\max }(T)$.

Main result.-We can now formulate the main result.

Observation 1 (entanglement cannot enhance classical capacity of noisy quantum channels to its maximum value).-Every quantum channel that is noisy (in the sense that the single-shot classical capacity is not the maximum output entropy) cannot be made to have maximum capacity with the help of any sophisticated entangled input.

So if there is a gap to the maximum possible single-shot capacity, this gap will be preserved in the asymptotic limit, independent of $n$ : No entangled input can overcome this limitation. The single-shot classical capacity may be nonadditive, as has been shown in Ref. [1]. Yet, entanglement can only help to some extent and can, in particular, not make any imperfect channel perfect.

Upper bounds for classical capacities.-In order to show this result and the equivalent one for the quantum capacity, we make use of upper bounds to channel capacities, starting with the classical capacity. The bounds forming the tools of the argument will be provided by quantities that capture the entanglement between a system and its environment in a dilation of the channel. We first show what properties a general quantity $M: \mathcal{S}\left(\mathbb{C}^{d} \otimes\right.$ $\left.\mathbb{C}^{d_{\text {env }}}\right) \rightarrow \mathbb{R}^{+}$, defined on bipartite quantum systems, should have. In order to be entirely clear, we will always give the tensor factors with respect to which an entanglement measure will be taken. For example, $M(\sigma, A: B)$ would be the quantity evaluated for $\sigma$ with respect to the split $A: B$. Two properties will be important. (1) $M$ has the property that

$$
E_{F}(\sigma, A: B) \geq \sum_{j=1}^{n} M\left(\sigma_{A_{j}, B_{j}}, A_{j}: B_{j}\right)
$$

for every bipartite state $\sigma$ defined on $n$ copies of a bipartite quantum system, labeled $B_{1}, \ldots, B_{n}$ and $A_{1}, \ldots, A_{n}, \sigma_{A_{j}, B_{j}}$ denoting the respective reduction. Here, $E_{F}$ denotes the entanglement of formation [3]. (2) $M$ is faithful. That is, $M(\rho, A: B)>0$ for bipartite states $\rho$ on $A$ and $B$ if and only if $\rho$ is entangled with respect to this split. As it turns out, for any quantity satisfying property 1 , the following bound holds true:

Observation 2 (upper bound for the classical capacity).- - For any quantum channel $T$ and any quantity $M$ satisfying condition 1 , we find the single-shot upper bound

$$
C(T) \leq \max _{\rho}\left\{S[T(\rho)]-M\left(U \rho U^{\dagger}, B: E\right)\right\} .
$$

The argument leading to this bound is remarkably simple: Starting from Eq. (2), and defining $\sigma=U^{\otimes n} \rho\left(U^{\otimes n}\right)^{\dagger}$ with reductions $\sigma_{B_{1}, \ldots, B_{n}}=\operatorname{tr}_{E_{1}, \ldots, E_{n}}(\sigma), B$ being formed by $B_{1}, \ldots, B_{n}$ and $E$ by $E_{1}, \ldots, E_{n}$, we find, using the Matsumoto-Shimono-Winter correspondence [9],

$$
\begin{aligned}
\chi\left(T^{\otimes n}\right) & =\max _{\rho}\left\{S\left[T^{\otimes n}(\rho)\right]-E_{F}\left[U^{\otimes n} \rho\left(U^{\otimes n}\right)^{\dagger}, B: E\right]\right\} \\
& =\max _{\rho}\left[S\left(\sigma_{B_{1}, \ldots, B_{n}}\right)-E_{F}(\sigma, B: E)\right] \\
& \leq \max _{\rho}\left(\sum_{j=1}^{n} S\left(\sigma_{B_{j}}\right)-E_{F}(\sigma, B: E)\right),
\end{aligned}
$$

using subadditivity, and hence, using property 1 ,

$$
\begin{aligned}
\chi\left(T^{\otimes n}\right) & \leq \max _{\rho} \sum_{j=1}^{n}\left[S\left(\sigma_{B_{j}}\right)-M\left(\sigma_{B_{j}, E_{j}}, B_{j}: E_{j}\right)\right] \\
& \leq \underset{\rho}{\max }\left\{S[T(\rho)]-M\left(U \rho U^{\dagger}, B: E\right)\right\},
\end{aligned}
$$

which is the above single-shot bound of Observation 2 .

This bound is to be compared with the MatsumotoShimono-Winter expression [9] for the Holevo- $\chi$ itself,

$$
\chi(T)=\max _{\rho}\left\{S[T(\rho)]-E_{F}\left(U \rho U^{\dagger}, B: E\right)\right\} .
$$

This is very similar, except that now the entanglement of formation takes the role of the quantity $M$. This indeed leads also to the conclusion of Observation 1 for the classical capacity: $C(T)$ achieves the maximum upper bound $\max _{\rho} S[T(\rho)]$ if and only if $\chi(T)$ achieves it. This is because 
$\chi$ achieves it if and only if $E_{F}\left(U \rho U^{\dagger}, B: E\right)=0$ for the maximizing $\rho$ in $\max _{\rho} S[T(\rho)]$, which means that $U \rho U^{\dagger}$ has to be separable. Now, if $M$ is also faithful, i.e., it satisfies property 2, then we can see that also $C$ achieves $\max _{\rho} S[T(\rho)]$ iff the optimal $U \rho U^{\dagger}$ is separable [10], which proves Observation 1. Below we shall provide a list of quantities, most of them satisfying both of the postulates.

Identifying candidates for suitable entanglement measures. - This result, needless to say, leaves the question of finding entanglement measures exhibiting the above properties 1 and 2. In other words, we need at least one such measure to prove the claim. Moreover, any computable measure satisfying property 1 will give rise to a useful bound for capacity.

(a) The entanglement measure G.-Define as in Ref. [12]

$$
C \leftarrow(\rho, B: E)=S\left(\rho_{B}\right)-\inf \sum_{i=0}^{k-1} q_{i} S\left(\frac{\operatorname{tr}_{E}\left[\left(\mathbb{1} \otimes P_{i}\right) \rho\left(\mathbb{1} \otimes P_{i}\right)^{\dagger}\right]}{q_{i}}\right),
$$

where the infimum is performed over all Kraus operators $P_{0}, \ldots, P_{k-1}$ acting on $E$ only, satisfying $\sum_{i=0}^{k-1} P_{i}^{\dagger} P_{i}=\mathbb{1}$, and $q_{i}=\operatorname{tr}\left[\left(\mathbb{1} \otimes P_{i}\right) \rho\left(\mathbb{1} \otimes P_{i}\right)^{\dagger}\right]$. This is a computable single-shot quantity. We denote the convex hull of this function with $G$ :

$$
G(\rho, B: E)=\min \sum_{j} p_{j} C_{\leftarrow}\left(\rho_{j}, B: E\right),
$$

where $\rho=\sum_{j} p_{j} \rho_{j}$, and which is an "entanglement measure" in its own right (it is at least a monotone under oneway LOCC [13]). We claim that this function has the right properties.

Observation 3 (bounding capacities in terms of classical correlations). - The quantity $G$ has the properties 1 and 2 .

Let us first check property 2: Every separable state will have a convex combination in terms of products, for each of which $C_{\leftarrow}$ will vanish. In turn, if a state is entangled, then there must in any convex combination be at least an entangled and hence correlated term, which will be detected by $C_{\leftarrow}$. To show property 1 , we can make use of a result of Ref. [11]: For a pure tripartite state $\rho$ shared by $A$, $B$, and $C$, a duality relation gives rise to $S\left(\rho_{A}\right)=$ $E_{F}\left(\rho_{A, B}, A: B\right)+C_{\leftarrow}\left(\rho_{A, C}, A: C\right)$. We now use the steps of Ref. [11] iteratively. For a mixed four-partite state $\rho$ on $A$, $B, C$, and $D$, the optimal decomposition for $E_{F}(\rho, A B: C D)$ in terms of pure states being $\left\{p_{j}, \rho_{j}\right\}$, for each $\rho_{j}$ we have $S\left(\rho_{j ; A, B}\right)=E_{F}\left(\rho_{j ; A, B, C}, A B: C\right)+C \leftarrow\left(\rho_{j ; A, B, D}, A B: D\right) \geq$ $E_{F}\left(\rho_{j ; A, C}, A: C\right)+C_{\leftarrow}\left(\rho_{j ; B, D}, B: D\right)$. Hence

$$
\begin{aligned}
E_{F}(\rho, A B: C D) & \geq \sum_{j} p_{j}\left[E_{F}\left(\rho_{j ; A, C}, A: C\right)+C \leftarrow\left(\rho_{j ; B, D}, B: D\right)\right] \\
& \geq E_{F}\left(\rho_{A, C}, A: C\right)+G\left(\rho_{B, D}, B: D\right),
\end{aligned}
$$

arriving at property 1 . This gives rise to a computable bound. Explicitly, it reads

$$
C(T) \leq \max _{\left\{p_{j}, \rho_{j}\right\}}\left\{S[T(\rho)]-\sum_{j} p_{j} C_{\leftarrow}\left(U \rho_{j} U^{\dagger}, B: E\right)\right\},
$$

with $\rho=\sum_{j} p_{j} \rho_{j}$, as a single maximization. An upper bound to this is

$$
C(T) \leq \max _{\rho} S[T(\rho)]-\min _{\rho} C_{\leftarrow}\left(U \rho U^{\dagger}, B: E\right),
$$

which is usually less tight but much simpler to compute.

(b) Variants of the relative entropy of entanglement.The measure proposed in Ref. [14] is superadditive and not larger than the entanglement of formation, implying property 1 . It is also shown to be faithful in Ref. [14].

(c) Squashed entanglement. - The squashed entanglement [15] $E_{\mathrm{sq}}$ is also known to be superadditive and is bounded from above by the entanglement of formation, so it qualifies as a bound for the same reason. It is not easily computable, however, as it is based on a construction involving a state extension the dimension of which is not bounded. However, a lower bound to squashed entanglement was provided in Ref. [16]:

$$
E_{\mathrm{sq}}\left(U \rho U^{\dagger}, B: E\right) \geq \frac{1}{4 \ln (2) d d_{\mathrm{env}}}\left(\min _{\sigma}\left\|U \rho U^{\dagger}-\sigma\right\|_{1}\right)^{2},
$$

in terms of the trace-norm distance to the set of separable quantum states $\sigma$ with respect to the split $B: E$.

(d) Distillable entanglement.-A not efficiently computable but in instances practical bound is provided by the distillable entanglement with respect to $B: E$, or so-called PPT-distillable entanglement [17]. (Note that either version of distillable entanglement does not satisfy property 2.)

Example: The amplitude qubit damping channel.-To find any nontrivial bound for the capacity of the amplitudedamping channel has been an open problem for some time [19]. The methods proposed here give rise to such bounds. The Kraus operators of $T(\rho)=K_{0} \rho K_{0}^{\dagger}+K_{1} \rho K_{1}^{\dagger}$ are given by

$$
K_{0}=\sqrt{p}|0\rangle\left\langle 1\left|, \quad K_{1}=\right| 0\right\rangle\langle 0|+\sqrt{1-p}| 1\rangle\langle 1|
$$

for $p \in[0,1]$. The isometry $U$ of this qubit channel maps $|0\rangle \mapsto|0,0\rangle,|1\rangle \mapsto \sqrt{p}|0,1\rangle+\sqrt{1-p}|1,0\rangle$. To bound the correlation measure $C_{\leftarrow}\left(U \rho U^{\dagger}, B: E\right)$, any choice for $k$ and for $P_{0}, \ldots, P_{k-1}$ giving rise to a positive operator valued measure amounts to a valid bound. This gives rise to the bound depicted in Fig. 2 [20] for $p \in[0,1 / 2]$. Note that it is significantly tighter than the trivial bound $C(T) \leq$ $S_{\max }(T)$, which here takes the value 1 . It is easy to see that for $p \in[0,1 / 2]$ there always exists an input diagonal in the computational basis that yields an output $\operatorname{diag}(1,1) / 2$ with unit entropy. For $p=0$ the channel becomes the perfect channel with $C(T)=1$. (The entanglement assisted classical information capacity [21] is also a crude upper bound but yields values even larger than 1 for $p \in[0,1 / 2])$. We have hence established a first nontrivial bound for the amplitude-damping channel. Needless to say, the same techniques can be applied to any finitedimensional quantum channel.

Quantum capacity.-It turns out that the quantum capacity has the same feature as the classical capacity. Again, entanglement can help to a certain degree but never uplift 


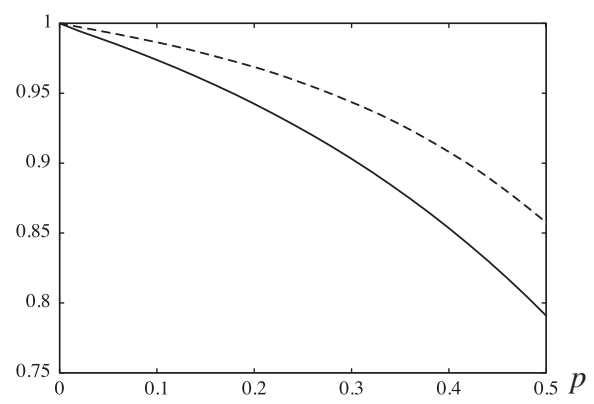

FIG. 2. Upper bound to the classical information capacity of the qubit amplitude-damping channel as a function of $p \in[0,1 / 2]$. The chosen Kraus operators delivering good bounds for $k=2$ are given by $P_{0}=|0\rangle\left\langle 0\left|/ 2, P_{1}=\sum_{i, j=0,1}\right| i\right\rangle \times$ $\langle j| / x, P_{2}=\left(\mathbb{1}-P_{0}^{\dagger} P_{0}-P_{1}^{\dagger} P_{1}\right)^{1 / 2}$ for $x=4$ (dashed line) and $x=3$ (solid line). Any choice for Kraus operators yields a bound, and with the given choice (determined via numerical explorations) one obtains a particularly tight bound.

channels to the maximum possible value. We arrive at the following conclusion (see Ref. [22] for the proof).

Observation 4 (entanglement cannot enhance the quantum capacity to its maximum value).-For every quantum channel for which the single-shot quantum capacity is not yet already given by the trivial upper bound $S_{\max }(T)$, the same will hold true for the quantum capacity.

Summary and outlook.-In this work, we have investigated the converse question to the additivity problem: How much can entanglement help enhance capacities of quantum channels? In the focus of interest was the question of whether entanglement can ever enhance the capacity to its trivial maximum if a single invocation does not yet reach that. We affirmatively answer that question to the negative, including the quantum and classical capacity. In doing so, we have established practical computable upper bounds to classical capacity, relating it to entanglement measures and rendering bounds and witnesses to the latter quantities useful to assess the classical capacity. There is though an interesting challenge: All the quantities from our list exhibit a sort of monogamy; i.e., for states which are highly sharable, they have to be small, implying that the bounds may become loose. An example is a channel whose Stinespring dilation gives rise to a $d$-dimensional antisymmetric space. A normalized projector onto this subspace is $d$-sharable, which means that for large $d$ all our bounds would tend to $\log _{2} d$, while a direct approach of Ref. [23] shows that the capacity is bounded by a constant independent of $d$. An open question is therefore how to find a quantity satisfying our property 1 , but that would not necessarily drop for sharable states. It is the hope that the present work triggers further work on how "small" violations of additivity really are in practice and what role entanglement plays after all in quantum communication.

We thank M. Christandl, A. Harrow, M. P. Müller, and A. Winter for useful feedback. F. G. S. L. B. is supported by the Brazilian agency Fundação de Amparo a Pesquisa do Estado de Minas Gerais (FAPEMIG). J. E. is supported by the EU (QESSENCE, MINOS, COMPAS), the BMBF (QuOReP), and the EURYI. M.H. is supported by MNiSW Grant No. N202 231937 and by the EU (QESSENCE). D. Y. is supported by NSFC (Grant No. 10805043). Part of this work was done in KCIK. F. G. S. L. B., J. E., and M. H. are thankful for the hospitality of the Mittag-Leffler institute, where part of this work has been done.

[1] M. Hastings, Nature Phys. 5, 255 (2009).

[2] C. King and M. B. Ruskai, IEEE Trans. Inf. Theory 47, 192 (2001); C. King, J. Math. Phys. (N.Y.) 43, 4641 (2002); C. King, Quantum Inf. Comput. 3, 186 (2003); M. Fannes et al. arXiv:quant-ph/0410195; P. W. Shor, J. Math. Phys. (N.Y.) 43, 4334 (2002); R. Alicki and M. Fannes, Open Syst. Inf. Dyn. 11, 339 (2004); M. M. Wolf and J. Eisert, New J. Phys. 7, 93 (2005).

[3] R. Horodecki, P. Horodecki, and M. Horodecki, Rev. Mod. Phys. 81, 865 (2009).

[4] G. G. Amosov, A. S. Holevo, and R. F. Werner, Probl. Inf. Transm. 36, 25 (2000).

[5] P. W. Shor, Commun. Math. Phys. 246, 453 (2004); K. M. R. Audenaert and S. L. Braunstein, ibid. 246, 443 (2004).

[6] P. Hayden and A. Winter, Commun. Math. Phys. 284, 263 (2008).

[7] P. W. Shor and J. A. Smolin, quant-ph/9604006.

[8] A.S. Holevo, arXiv:quant-ph/9809023.

[9] K. Matsumoto, T. Shimono, and A. Winter, Commun. Math. Phys. 246, 427 (2004).

[10] This result may indeed be viewed as the channel analogue of the observation that the entanglement cost $E_{C}$ is strictly positive for every entangled state [11].

[11] D. Yang et al., Phys. Rev. Lett. 95, 190501 (2005).

[12] L. Henderson and V. Vedral, J. Phys. A 34, 6899 (2001).

[13] "LOCC" stands for local operations and classical communication. We say "one-way LOCC" if the communication is allowed only in one direction (here from $E$ to $B$ ).

[14] M. Piani, Phys. Rev. Lett. 103, 160504 (2009).

[15] M. Christandl and A. Winter, J. Math. Phys. (N.Y.) 45, 829 (2004); R. R. Tucci, arXiv:quant-ph/9909041.

[16] F. G.S.L. Brandao, M. Christandl, and J. Yard, arXiv: 1010.1750.

[17] For the definition of PPT-distillable entanglement, see Ref. [18].

[18] E. M. Rains, IEEE Trans. Inf. Theory 47, 2921 (2001); K. M. R. Audenaert et al., Phys. Rev. Lett. 87, 217902 (2001).

[19] D. Leung and G. Smith (private communication).

[20] This is numerically evaluated by optimizing over mixedstate ensembles $\rho=\sum_{j} p_{j} \rho_{j}$ using global optimization both based on the routine FMINCON in MATLAB with randomly sampled initial conditions and simulated annealing.

[21] C. H. Bennett et al., arXiv:quant-ph/0106052.

[22] See supplemental material at http://link.aps.org/ supplemental/10.1103/PhysRevLett.106.230502 for the proof of Observation 4.

[23] M. Christandl, N. Schuch, and A. Winter, Phys. Rev. Lett. 104, 240405 (2010). 